\title{
Racial and Ethnic Differences in Mortality Associated with Serum Potassium in a Large Hemodialysis Cohort
}

\author{
Taehee Kim ${ }^{a, e}$ Connie M. Rhee ${ }^{a}$ Elani Streja ${ }^{a}$ b Melissa Soohoo ${ }^{a}$ \\ Yoshitsugu Obi ${ }^{\mathrm{a}}$ Jason A. Chou ${ }^{\mathrm{a}}$ Amanda R. Tortorici $^{\mathrm{a}}$ Vanessa A. Ravel ${ }^{\mathrm{a}}$ \\ Csaba P. Kovesdy ${ }^{c, d}$ Kamyar Kalantar-Zadeha, b \\ ${ }^{a}$ Harold Simmons Center for Kidney Disease Research and Epidemiology, University of California Irvine, School \\ of Medicine, Orange, CA, ${ }^{b}$ Nephrology Section, Tibor Rubin Veterans Affairs Medical Center, Long Beach, CA, \\ and 'Nephrology Section, Memphis Veterans Affairs Medical Center, and d Division of Nephrology, University of \\ Tennessee Health Science Center, Memphis, TN, USA; ${ }^{e}$ Department of Medicine, Inje University, Busan, South Korea
}

\section{Keywords}

Potassium · Hemodialysis · Chronic kidney disease · Mortality

\begin{abstract}
Background: Hyperkalemia is observed in chronic kidney disease patients and may be a risk factor for life-threatening arrhythmias and death. Race/ethnicity may be important modifiers of the potassium-mortality relationship in maintenance hemodialysis (MHD) patients given that potassium intake and excretion vary among minorities. Methods: We examined racial/ethnic differences in baseline serum potassium levels and all-cause and cardiovascular mortality using Cox proportional hazard models and restricted cubic splines in a cohort of 102,241 incident MHD patients. Serum potassium was categorized into 6 groups: $\leq 3.6,>3.6$ to $\leq 4.0,>4.0$ to $\leq 4.5$ (reference), $>4.5$ to $\leq 5.0,>5.0$ to $\leq 5.5$, and $>5.5 \mathrm{mEq} / \mathrm{L}$. Models were adjusted for case-mix and malnutrition-inflammation cachexia syndrome (MICS) covariates. Results: The cohort was composed of 50\% whites, 34\% African-Americans, and $16 \%$ Hispanics. Hispanics tended to have the highest baseline serum potassium levels (mean \pm SD: $4.58 \pm 0.55$
\end{abstract}

\section{KARGER}

(c) 2017 S. Karger AG, Basel

E-Mail karger@karger.com

www.karger.com/ajn
$\mathrm{mEq} / \mathrm{L})$. Patients in our cohort were followed for a median of 1.3 years (interquartile range $0.6-2.5$ ). In our cohort, associations between higher potassium $(>5.5 \mathrm{mEq} / \mathrm{L})$ and higher mortality risk were observed in African-American and whites, but not Hispanic patients in models adjusted for case-mix and MICS covariates. While in Hispanics only, lower serum potassium $(<3.6 \mathrm{mEq} / \mathrm{L})$ levels were associated with higher mortality risk. Similar trends were observed for cardiovascular mortality. Conclusions: Higher potassium levels were associated with higher mortality risk in white and AfricanAmerican MHD patients, whereas lower potassium levels were associated with higher death risk in Hispanics. Further studies are needed to determine the underlying mechanisms for the differential association between potassium and mortality across race/ethnicity.

(C) 2017 S. Karger AG, Basel

\section{Introduction}

The kidneys play a primary role in the regulation of potassium homeostasis by balancing potassium intake with excretion [1]. When kidney function declines, dis- 
ruption in homeostasis and subsequent hyperkalemia are commonly observed, and the latter may be ameliorated by a reduction of potassium intake and utilization of potassium-lowering medications that augment potassium excretion via the gastrointestinal tract [2]. Despite these interventions, hyperkalemia is more frequently observed in chronic kidney disease (CKD) patients compared to the non-CKD population [3]. Dissecting the potassiummortality relationship is of particular importance, given that maintenance hemodialysis (MHD) patients have an exceedingly high cardiovascular mortality $(40 \%$ of all deaths) largely due to sudden cardiac arrest, and hyperkalemia may lead to life-threatening cardiac arrhythmias and higher death risk in this population [4-6].

Several studies suggest that there may be differences in potassium homeostasis across racial/ethnic groups. For example, prior data has shown that dietary potassium intake and/or urinary potassium excretion used to determine serum potassium levels differs in whites and African-Americans [7, 8]. It is also well-established that there are racial/ethnic differences in the mortality of maintenance dialysis patients, such that African-Americans have a survival advantage compared to white patients, particularly in those of middle and older age groups [9-11]. While there have been fewer studies in Hispanic MHD patients, emerging data suggests that Hispanics also have a survival advantage compared to non-Hispanic whites and African-Americans [11-13], even after accounting for differences in socioeconomic factors and comorbid conditions [14]. Whereas epidemiologic data show that serum potassium levels differ across race/ethnicity in the general population, there has been no prior study on the distribution of serum potassium levels and their associations with mortality across race/ethnicity in MHD patients [8]. Therefore, we examined racial/ethnic differences in serum potassium levels across whites, AfricanAmericans, and Hispanics in a contemporary cohort of incident MHD patients who were followed up to 5 years. Moreover, we examined whether there is a differential association between serum potassium level and mortality risk across these racial/ethnic groups.

\section{Materials and Methods}

\section{Source Population}

We conducted analyses using administrative data from all incident hemodialysis (HD) patients who initiated dialysis from January 1, 2007 to December 31, 2011 in the outpatient facilities of the large dialysis organization. From January 2007 to December 2011 , there were 208,820 patients who initiated dialysis treatment.
After excluding patients less than 18 years of age, patients who received less than 60 days of dialysis treatment, or were ever treated with any other dialysis modality, 133,156 incident HD patients remained.

After excluding patients who were not of white, AfricanAmerican, and Hispanic race/ethnicity and those with missing baseline serum potassium information, the final study population consisted of 102,241 incident HD patients (online suppl. Appendix-Fig. S1; for all online suppl. material, see www.karger.com/ doi/10.1159/000475997). The study was approved by the International Review Committees of the University of California Irvine, Los Angeles Biomedical Research Institute at Harbor-UCLA, and the University of Washington. Given the large sample size, anonymity of the patients studied, and nonintrusive nature of the research, the requirement for written consent was exempted.

\section{Socio-Demographic and Clinical Measures}

Over $99 \%$ of the cohort had information on race/ethnicity which was based on "self-identified" data based on definitions of the US Census Bureau and guidance of US Office of Management and Budget. Information on primary insurance, initial vascular access type, and the presence of comorbidities at baseline were obtained from the large dialysis organization database. The following coexisting comorbidities were also considered: diabetes mellitus (DM), hypertension, congestive heart disease (CHF), atherosclerotic heart disease, other cardiac disease (pericarditis and cardiac arrhythmia), cerebrovascular disease, chronic obstructive pulmonary disease, liver disease, dyslipidemia, malignancy, human immunodeficiency virus antibody positive status, substance abuse, and alcohol abuse.

\section{Laboratory and Clinical Measures}

Blood samples were drawn using standardized techniques in all dialysis clinics and were transported to a central laboratory in Deland, Florida, typically within $24 \mathrm{~h}$ of collection. Most laboratory values were measured monthly, including serum potassium, creatinine, albumin, hemoglobin, platelet count, peripheral white blood cell count (WBC), lymphocyte percentage, total iron binding capacity (TIBC), calcium, phosphorus, bicarbonate, blood urea nitrogen (BUN), and alkaline phosphatase. Serum intact parathyroid hormone (iPTH), and ferritin levels were usually measured at least once during each calendar quarter. Most blood samples were collected before $\mathrm{HD}$, except for post-dialysis BUN to calculate urea kinetics. The normalized protein catabolic rate (nPCR) was measured monthly as an indicator of daily protein intake. Dialysis dose was estimated by single pool Kt/V (spKt/V) using the urea kinetic model. The average serum urea concentrations during the collection were assumed to be $90 \%$ of the pre-dialysis concentration according to the Daugirdas approach, and thus residual renal function was calculated as follows [15].

$$
\begin{aligned}
& \text { urinary urea }(\mathrm{mg} / \mathrm{dL}) \times \\
& \text { Residual renal function }(\mathrm{mL} / \mathrm{min})=\frac{\text { urinary volume }(\mathrm{mL})}{\text { collected time }(\mathrm{min}) \times} \\
& {[0.9 \times \text { serum urea }(\mathrm{mg} / \mathrm{dL})]}
\end{aligned}
$$

The residual renal function was adjusted for body surface area and expressed as $\mathrm{mL} / \mathrm{min} / 1.73 \mathrm{~m}^{2}[16,17]$. Ultrafiltration (UF) was calculated as the difference between pre-HD weights and post- 
HD weights that were measured every HD session. Seated pre-HD blood pressure (BP) values were measured every HD session. The median weekly erythropoiesis stimulating agent (ESA) dose was calculated by dividing the cumulative quarterly ESA dose by the total number of HD treatments over a 91-day period, and then multiplying the per-treatment dose by the number of median treatments per week. To minimize measurement variability, all repeated measures for each patient during any given patient quarter (i.e., 91-day intervals) were averaged, and summary estimates used in all analyses. The 91-day averaged values during the first patient quarter of dialysis treatment were used as baseline values.

\section{Outcome Measures}

The recorded causes of death were obtained from the large dialysis organization data records, and cardiovascular death was defined as death due to cardiovascular reasons, including acute myocardial infarction, cardiac arrest, heart failure, cerebrovascular accident, and other cardiac disease. Patients were followed from 91 days from the start of dialysis to death, kidney transplantation, transfer to a non-affiliated dialysis unit, recovery of renal function, dialysis discontinuation or end of the study period (December 31, 2011).

\section{Statistical Methods}

Baseline characteristics across race/ethnicity groups were summarized as proportions, mean $\pm \mathrm{SD}$, or medians (interquartile ranges) depending on data type. We used logistic regression to estimate the association between clinical characteristics with the likelihood of hyperkalemia (serum potassium $>5.0$ vs. $>3.6$ to $\leq 5.0$ $\mathrm{mEq} / \mathrm{L}$ ) or hypokalemia ( $\leq 3.6$ vs. $>3.6$ to $\leq 5.0 \mathrm{mEq} / \mathrm{L}$ ) across racial/ethnic groups.

We examined the association between serum potassium as a continuous variable using restricted cubic spline analyses (with knots at the 33rd and 66th percentile for each group's potassium distribution) and all-cause mortality across racial/ethnic groups using Cox proportional hazards model. We then stratified patients according to the 6 groups of baseline serum potassium representing a combination of clinical reference ranges and the distribution of our cohort $(\leq 3.6,>3.6$ to $\leq 4.0,>4.0$ to $\leq 4.5,>4.5$ to $\leq 5.0,>5.0$ to $\leq 5.5$, and $>5.5 \mathrm{mEq} / \mathrm{L}$ ) and the $3 \mathrm{racial} /$ ethnic groups (i.e., total of 18 categories), and examined their associations with mortality. We also separately examined the association of hyperkalemia and hypokalemia (reference: serum potassium $>3.6$ to $\leq 5.0 \mathrm{mEq} / \mathrm{L}$ ) with all-cause and cardiovascular mortality in the overall cohort and across clinically relevant subgroups stratified by race/ethnicity, and $p$ values for interactions between serum potassium and subgroup covariates were assessed. Proportional hazards assumptions were assessed by graphical methods.

For each analysis, 3 levels of multivariable adjustment were used: (1) unadjusted models that included the main predictor, serum potassium; (2) case-mix adjusted models that included covariates in the unadjusted model as well as age, sex, DM, primary insurance, initial vascular access type, spKt/V, HD treatment time, $\mathrm{UF}$, and cardiovascular risk factors including pre-HD systolic BP, pre-HD diastolic BP, body mass index (BMI), and previously mentioned comorbidities; (3) case-mix + malnutrition-inflammation cachexia syndrome (MICS) adjusted models that included covariates in the case-mix model as well as 12 surrogates of nutritional and/or inflammatory status: albumin, hemoglobin, peripheral WBC, lymphocyte percentage, ferritin, TIBC, calcium, phosphorus, bicarbonate, BUN, iPTH, nPCR, and ESA dose.

Potassium and Mortality across Race/ Ethnicity

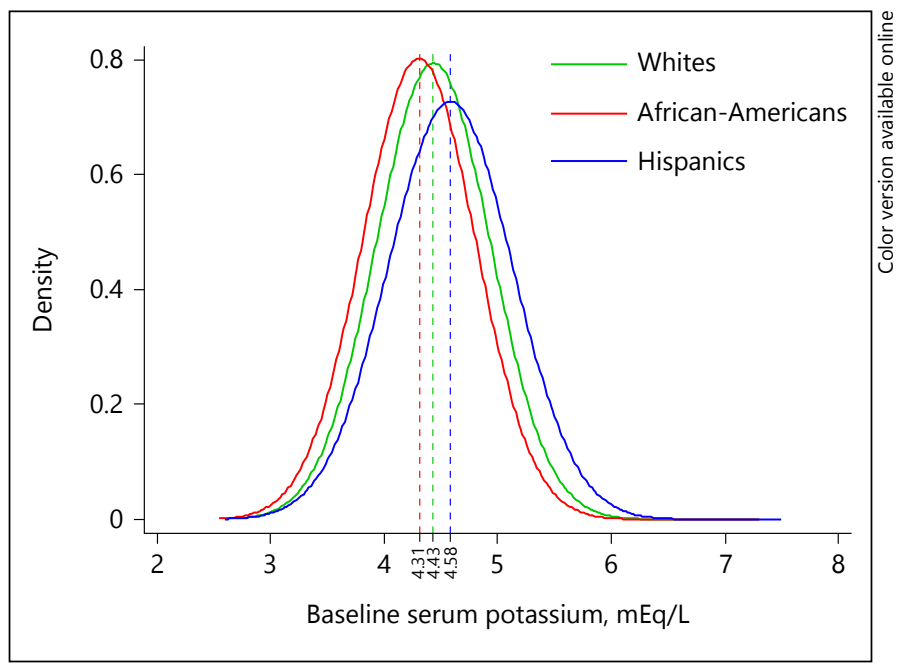

Fig. 1. Distribution of baseline serum potassium levels across whites, African-Americans, and Hispanics in 102,241 patients. Dashed lines show the mean potassium level of each race/ethnic group.

To account for the competing risk of transplantation across race/ethnicity in sensitivity analyses, we conducted Fine and Gray competing risk regression to estimate the sub-hazard ratios of death risk [18]. To address potential residual confounding on the basis of residual renal function, sensitivity analyses that additionally adjusted for residual urine volume were conducted. While there were no missing data for age, data for sex, race/ethnicity, and comorbidities, missing covariates $<5 \%$ at study entry were imputed by mean or median values as appropriate. All analyses were conducted using STATA MP version 13.1 (StataCorp, College Station, TX, USA).

\section{Results}

\section{Study Population Description}

The mean \pm SD age of the overall cohort was $63 \pm 15$ years and the mean \pm SD serum potassium level was $4.4 \pm 0.5 \mathrm{mEq} / \mathrm{L}$. The median duration of follow-up was 1.3 years (25th-75th percentile, 0.6-2.5 years). The crude all-cause mortality rate of the overall cohort was 139 death events per 1,000 patient-years of follow-up (95\% CI 138-141 death events per 1,000 patient-years). Differences in serum potassium levels were evident across racial/ethnic groups $(p<0.001)$, as Hispanics tended to have the highest baseline serum potassium levels (mean \pm SD: $4.58 \pm 0.55 \mathrm{mEq} / \mathrm{L}$ ), whereas AfricanAmericans had the lowest levels (mean \pm SD: $4.31 \pm 0.50$ $\mathrm{mEq} / \mathrm{L}$; Fig. 1). Baseline characteristics of the cohort stratified by race/ethnicity and baseline serum potassium level are presented in Table 1. Across all racial/eth- 


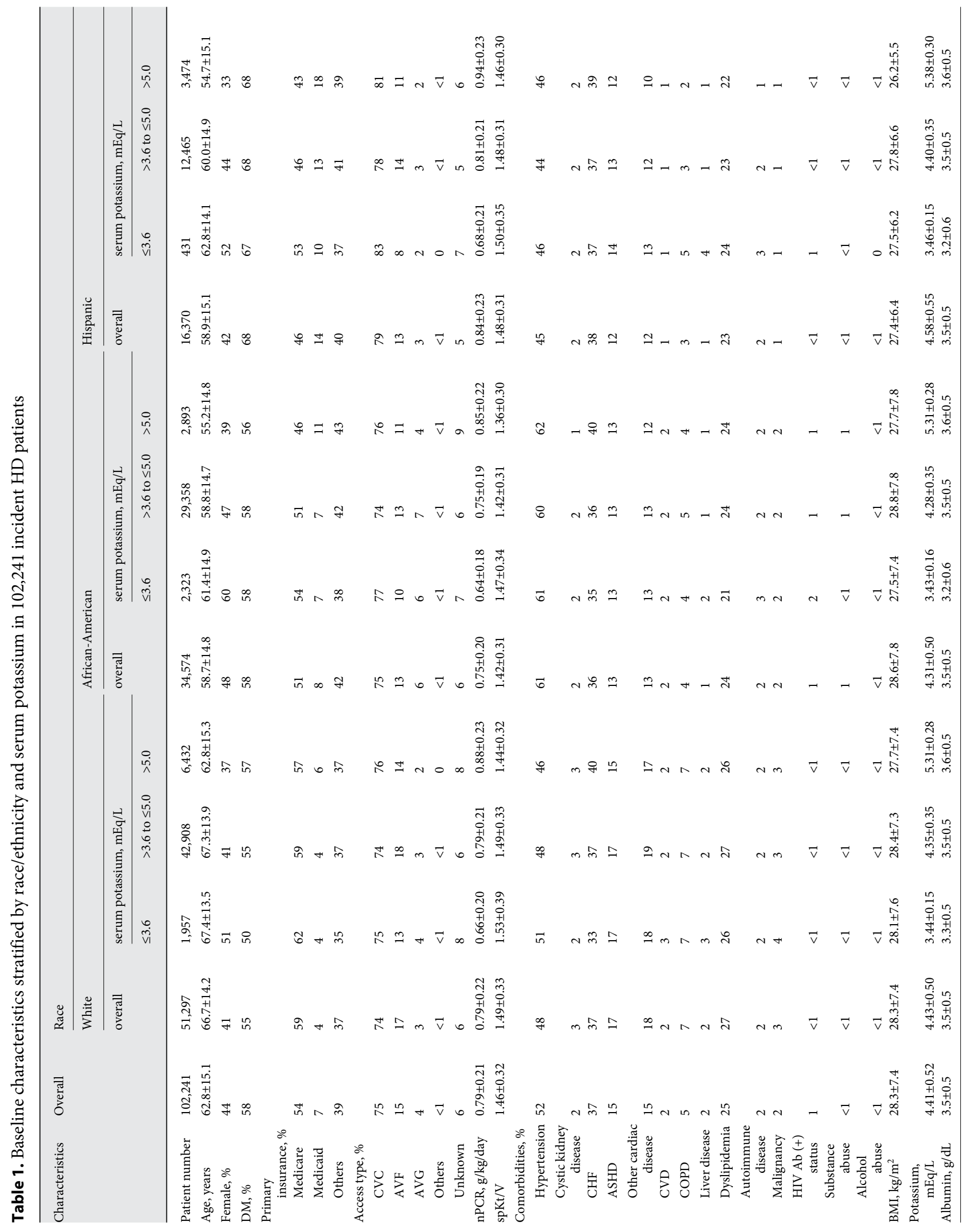




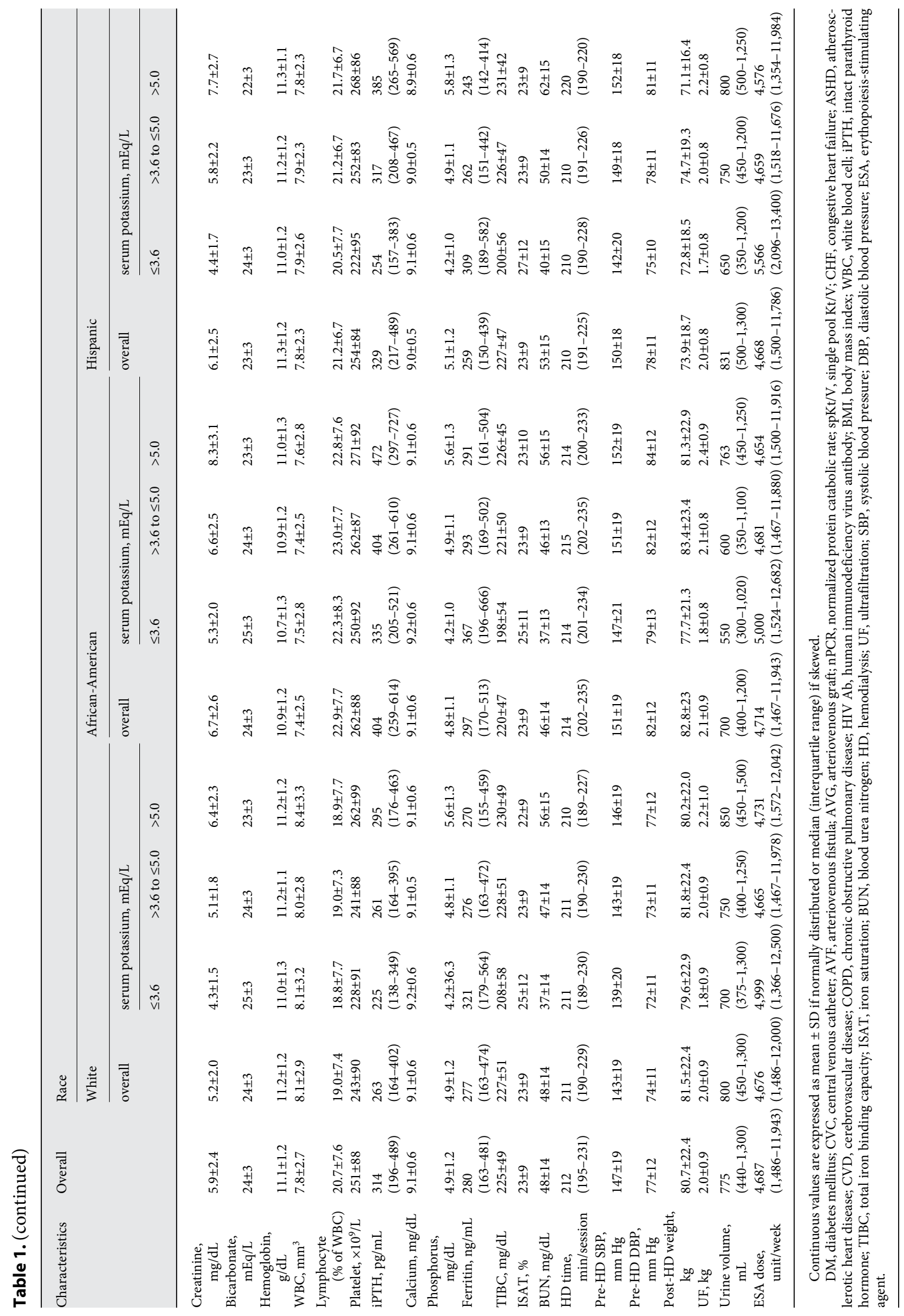


Table 2. Association of clinical characteristics with hyperkalemia (serum potassium $>5.0$ vs. $>3.6$ to $\leq 5.0$ mEq/L) among whites, AfricanAmericans, and Hispanics in case-mix + MICS adjusted logistic regression models

\begin{tabular}{|c|c|c|c|}
\hline Factors & \multicolumn{3}{|l|}{ Race } \\
\hline Age, $\Delta$ 10-year & $0.96(0.94-0.99)$ & $0.96(0.92-0.99)$ & $0.96(0.92-0.99)$ \\
\hline Female vs. male & $1.15(1.08-1.23)$ & $1.14(1.03-1.26)$ & $0.94(0.85-1.04)$ \\
\hline \multicolumn{4}{|l|}{ Comorbidity factors } \\
\hline Congestive heart failure & $1.06(1.00-1.12)$ & $1.10(1.01-1.20)$ & $1.05(0.96-1.15)$ \\
\hline $\mathrm{BMI}, \Delta 1 \mathrm{~kg} / \mathrm{m}^{2}$ & $0.97(0.96-0.97)$ & $0.96(0.96-0.97)$ & $0.95(0.94-0.95)$ \\
\hline \multicolumn{4}{|l|}{ Primary Insurance } \\
\hline Medicaid vs. Medicare & $1.07(0.93-1.22)$ & $1.31(1.13-1.51)$ & $1.09(0.96-1.23)$ \\
\hline Other vs. Medicare & $0.86(0.81-0.92)$ & $1.01(0.93-1.10)$ & $0.86(0.79-0.95)$ \\
\hline \multicolumn{4}{|l|}{ Dialysis-related factors } \\
\hline $\mathrm{spKt} / \mathrm{V}, \Delta 0.1$ unit & $0.97(0.96-0.98)$ & $0.97(0.95-0.99)$ & $0.97(0.95-0.99)$ \\
\hline HD time, $\Delta 10 \mathrm{~min} / \mathrm{session}$ & $0.99(0.98-1.00)$ & $0.97(0.96-0.99)$ & $0.97(0.96-0.99)$ \\
\hline Ultrafiltration, $\Delta 1 \mathrm{~kg}$ & $1.26(1.21-1.30)$ & $1.32(1.25-1.39)$ & $1.39(1.31-1.48)$ \\
\hline Pre-HD SBP, $\Delta 10 \mathrm{~mm} \mathrm{Hg}$ & $0.98(0.96-1.00)$ & $0.99(0.95-1.02)$ & $0.99(0.95-1.02)$ \\
\hline \multicolumn{4}{|l|}{ Nutritional factors } \\
\hline Albumin, $\Delta 0.1 \mathrm{~g} / \mathrm{dL}$ & $1.02(1.01-1.03)$ & $1.02(1.01-1.03)$ & $1.02(1.01-1.03)$ \\
\hline Phosphorus, $\Delta 0.1 \mathrm{mg} / \mathrm{dL}$ & $1.03(1.03-1.04)$ & $1.02(1.02-1.03)$ & $1.02(1.02-1.03)$ \\
\hline $\mathrm{BUN}, \Delta 1 \mathrm{mg} / \mathrm{dL}$ & $1.03(1.03-1.03)$ & $1.04(1.04-1.05)$ & $1.04(1.03-1.04)$ \\
\hline $\mathrm{nPCR}, \Delta 0.1 \mathrm{~g} / \mathrm{kg} /$ day & $1.00(0.98-1.02)$ & $0.96(0.93-1.00)$ & $0.96(0.93-1.00)$ \\
\hline
\end{tabular}

Case-mix + MICS models adjusted for age, sex, DM, primary insurance, vascular access type, spKt/V, HD treatment time, UF, and cardiovascular risk factors including pre-HD systolic BP, pre-HD diastolic BP, BMI, comorbidities, 13 surrogates of nutritional and/or inflammatory status (albumin, hemoglobin, peripheral WBC, lymphocyte percentage, ferritin, TIBC, calcium, phosphorus, bicarbonate, BUN, iPTH, nPCR, and ESA dose).

DM, diabetes mellitus; BMI, body mass index; AVF, arteriovenous fistula; CVC, central venous catheter; AVG, arteriovenous graft; spKt/V, single pool Kt/V; HD, hemodialysis; SBP, systolic blood pressure; BUN, blood urea nitrogen; nPCR, normalized protein catabolic rate.

nic groups, patients in the highest serum potassium category were more likely to be younger and less likely to use Medicare as their primary insurance. They were also more likely to have CHF and higher nPCR, albumin, creatinine, platelet, $\mathrm{iPTH}$, phosphorus, $\mathrm{BUN}$, pre-HD SBP and DBP, and UF levels; they had lower spKt/V and bicarbonate levels compared to those in the lowest potassium category.

\section{Clinical Characteristics Associated with Potassium \\ Level across Racial/Ethnic Groups}

In logistic regression analyses in the overall cohort, compared to white patients, African-Americans had a $42 \%$ lower odds of hyperkalemia (OR 0.58 , 95\% CI $0.55-$
0.62 ) and a $63 \%$ higher odds of hypokalemia (OR 1.63, 95\% CI 1.52-1.76), while Hispanic patients had a $32 \%$ higher odds of hyperkalemia (OR 1.32, 95\% CI $1.25-$ 1.39 ) and $11 \%$ lower odds of hypokalemia (OR 0.89, 95\% CI 0.80-0.99) in case-mix + MICS adjusted models in the overall cohort. Table 2 shows the association of clinical characteristics with the likelihood of hyperkalemia (reference: serum potassium $>3.6$ to $\leq 5.0 \mathrm{mEq} / \mathrm{L}$ ) across racial/ethnic groups in case-mix + MICS adjusted logistic regression models. Across all racial/ethnic groups, older age, higher baseline spKt/V, AV fistula access, and higher baseline BMI were associated with lower likelihood of hyperkalemia. Higher levels of nutritional status markers (higher albumin, phosphorus, and 
BUN), diabetes, higher UF, and shorter HD treatment time were associated with higher likelihood of hyperkalemia. Among both African-Americans and whites, females were more likely to have baseline hyperkalemia. In African-American patients only, Medicaid insurance type was associated with a higher likelihood of hyperkalemia.

In case-mix + MICS adjusted logistic regression models, clinical characteristics associated with a higher likelihood of hypokalemia (reference: serum potassium $>3.6$ to $\leq 5.0 \mathrm{mEq} / \mathrm{L}$ ), included lower serum albumin, phosphorus, and BUN across all racial/ethnic groups. Older age was a significant predictor of hypokalemia only among whites (OR [95\% CI] 0.88 [0.85-0.92]), while female sex was a predictor of hypokalemia (OR [95\% CI] 1.18 [1.061.31]) only in African-American patients (online suppl. Appendix-Table S1). There was no association between HD treatment time and hypokalemia for any racial/ethnic group.

\section{Serum Potassium and Mortality across Racial/ Ethnic Groups}

In case-mix + MICS adjusted cubic spline models, there was a J-shaped relationship between serum potassium and all-cause mortality in whites and AfricanAmericans, such that higher serum potassium levels were associated with higher mortality risk, while lower serum potassium levels had no significant association with mortality (Table 3 ). In whites, serum potassium levels $>4.5 \mathrm{mEq} / \mathrm{L}$ were associated with incrementally higher mortality risk in case-mix + MICS adjusted models (Fig. 2a): HRs [95\% CI] 1.05 [1.01-1.09], 1.09 [1.041.16], 1.17 [1.06-1.30] for serum potassium levels $>4.5$ to $\leq 5.0,>5.0$ to $\leq 5.5$, and $>5.5 \mathrm{mEq} / \mathrm{L}$, respectively. African-Americans also demonstrated an association between higher serum potassium level and higher mortality risk, albeit at a higher serum potassium threshold of $>5.5 \mathrm{mEq} / \mathrm{L}$ : HR (95\% CI) 1.43 (1.21-1.70) (Table 3).

Conversely, among Hispanics, lower serum potassium levels were associated with higher mortality, whereas higher serum potassium levels had no association with mortality in the case-mix + MICS adjusted models (Fig. 2a). In categorical analyses, only very low serum potassium levels $(\leq 3.6 \mathrm{mEq} / \mathrm{L})$ were significantly associated with higher mortality risk in Hispanics: HR (95\% CI) 1.36 (1.15-1.62) (Table 3). These trends were similar in the case-mix + MICS adjusted cubic spline models (online suppl. Appendix-Fig. S2). While serum potassium levels $>5.5 \mathrm{mEq} / \mathrm{L}$ trended towards higher cardiovascular mor-

Potassium and Mortality across Race/ Ethnicity tality risk in whites and African-Americans, they were associated with lower cardiovascular mortality risk in Hispanics, although this was not statistically significant (Fig. 2b).

Figure 3 shows the association between 18 categories of race/ethnicity and baseline serum potassium level with all-cause mortality. Compared to Hispanics with serum potassium levels $>4.0$ to $\leq 4.5 \mathrm{mEq} / \mathrm{L}$ as the reference category, all potassium categories were associated with higher mortality risk amongst whites in case-mix + MICS adjusted models. All potassium categories were associated with higher mortality risk amongst AfricanAmericans compared to the reference group. Amongst Hispanics, patients with serum potassium $>4.5 \mathrm{mEq} / \mathrm{L}$ had similar mortality risk compared to the reference group, whereas those with serum potassium $\leq 3.6 \mathrm{mEq} / \mathrm{L}$ had a higher mortality risk. Similar observations were observed after taking into consideration the impact of kidney transplantation as a competing event, in casemix + MICS adjusted models (online suppl. AppendixFig. S3).

\section{Potassium and Mortality across Subgroups}

Figure 4 shows the case-mix + MICS adjusted associations between hyperkalemia and all-cause mortality (reference: potassium levels $>3.6$ to $\leq 5.0 \mathrm{mEq} / \mathrm{L}$ ) across a priori selected subgroups stratified by race/ethnicity. Within white patients, there was effect modification on the basis of Medicare insurance type ( $p$-interaction $=0.007)$, and hypertension comorbidity $(p$-interaction $=0.004)$. There was no significant effect modification across subgroups among African-American or Hispanic patients. Similar associations of hypokalemia with mortality within each racial-ethnic group were persistent across most of all subgroups. In white patients, there was a significant effect modification by diabetes comorbidity $(p$-interaction $=$ 0.001 ) and in African-American by hypertension comorbidity ( $p$-interaction $=0.025)$. In both Hispanic and white patients, significant effect modification on the association of hypokalemia with mortality was observed ( $p$-interaction $\leq 0.001$ for each; online suppl. Appendix-Fig. S4).

To account for confounding on the basis of residual renal function, we incrementally adjusted the baseline urine volume within the case-mix + MICS adjusted models in a subcohort of 35,910 patients who had residual renal function data during the first patient quarter in sensitivity analyses. Although the potassium-mortality associations were attenuated across all racial/ethnic groups, trends of higher serum potassium level and higher mor- 
Table 3. The number of all-cause deaths, cardiovascular deaths, and transplantations across race/ethnicity and association between serum potassium and all-cause mortality according to race/ethnicity in case-mix and MICS adjusted Cox models

\begin{tabular}{|c|c|c|c|c|c|c|}
\hline \multirow[t]{3}{*}{ Serum potassium, $\mathrm{mEq} / \mathrm{L}$} & \multirow[t]{3}{*}{ Total, $n$} & \multicolumn{5}{|l|}{ Whites } \\
\hline & & \multirow[t]{2}{*}{ all-cause death, $n(\%)$} & \multirow[t]{2}{*}{ CV death, $n(\%)$} & \multirow[t]{2}{*}{ transplant, $n(\%)$} & \multicolumn{2}{|c|}{ all-cause mortality HR (95\% CI) } \\
\hline & & & & & unadjusted model & case-mix + MICS models \\
\hline Continuous value & 51,297 & $16,804(32.8)$ & $5,700(11.1)$ & $2,037(4.0)$ & $0.93(0.90-0.96)$ & $1.08(1.04-1.12)$ \\
\hline$\leq 3.6$ & 1,957 & $737(37.7)$ & $226(11.6)$ & $50(2.6)$ & $1.21(1.12-1.31)$ & $1.00(0.93-1.08)$ \\
\hline$>3.6$ to $\leq 4.0$ & 8,467 & $2,852(33.7)$ & $929(11.0)$ & $275(3.2)$ & $1.07(1.03-1.12)$ & $1.00(0.95-1.04)$ \\
\hline$>4.0$ to $\leq 4.5$ & 19,767 & $6,340(32.1)$ & $2,157(11.0)$ & $743(3.8)$ & 1 (reference) & 1 (reference) \\
\hline$>4.5$ to $\leq 5.0$ & 14,674 & $4,743(32.3)$ & $1,639(11.2)$ & $644(4.4)$ & $0.98(0.95-1.02)$ & $1.05(1.01-1.09)$ \\
\hline$>5.0$ to $\leq 5.5$ & 5,228 & $1,706(32.6)$ & $593(11.3)$ & $249(4.8)$ & $0.96(0.91-1.01)$ & $1.09(1.04-1.16)$ \\
\hline$>5.5$ & 1,204 & $426(35.4)$ & $156(13.0)$ & $76(6.3)$ & $1.01(0.91-1.11)$ & $1.17(1.06-1.30)$ \\
\hline \multirow[t]{3}{*}{ Serum potassium, $\mathrm{mEq} / \mathrm{L}$} & \multirow[t]{3}{*}{ total, $n$} & \multicolumn{5}{|l|}{ African-Americans } \\
\hline & & \multirow[t]{2}{*}{ all-cause death, $n(\%)$} & \multirow[t]{2}{*}{ CV death, $n(\%)$} & \multirow[t]{2}{*}{ transplant, $n(\%)$} & \multicolumn{2}{|c|}{ all-cause mortality HR (95\% CI) } \\
\hline & & & & & unadjusted model & case-mix + MICS models \\
\hline Continuous value & 34,574 & $7,573(21.9)$ & $2,654(7.7)$ & $878(2.5)$ & $0.81(0.77-0.85)$ & $1.00(0.95-1.05)$ \\
\hline$\leq 3.6$ & 2,323 & $707(30.4)$ & $213(9.2)$ & $42(1.8)$ & $1.51(1.39-1.64)$ & $1.07(0.98-1.17)$ \\
\hline$>3.6$ to $\leq 4.0$ & 7,642 & $1,780(23.3)$ & $628(8.2)$ & $172(2.3)$ & $1.13(1.07-1.20)$ & $1.01(0.95-1.07)$ \\
\hline$>4.0$ to $\leq 4.5$ & 13,767 & $2,884(20.9)$ & $1,011(7.3)$ & $340(2.5)$ & 1 (reference) & 1 (reference) \\
\hline$>4.5$ to $\leq 5.0$ & 7,949 & $1,571(19.8)$ & $563(7.1)$ & $227(2.9)$ & $0.92(0.86-0.97)$ & $0.98(0.92-1.04)$ \\
\hline$>5.0$ to $\leq 5.5$ & 2,384 & $491(20.6)$ & $192(8.1)$ & $85(3.6)$ & $0.93(0.85-1.02)$ & $1.01(0.92-1.12)$ \\
\hline$>5.5$ & 509 & $140(27.5)$ & $47(9.2)$ & $12(2.4)$ & $1.36(1.14-1.61)$ & $1.43(1.21-1.70)$ \\
\hline \multirow[t]{3}{*}{ Serum potassium, $\mathrm{mEq} / \mathrm{L}$} & \multirow[t]{3}{*}{ total, $n$} & \multicolumn{5}{|l|}{ Hispanics } \\
\hline & & \multirow[t]{2}{*}{ all-cause death, $n$ (\%) } & \multirow[t]{2}{*}{ CV death, $n(\%)$} & \multirow[t]{2}{*}{ transplant, $n(\%)$} & \multicolumn{2}{|c|}{ all-cause mortality HR (95\% CI) } \\
\hline & & & & & unadjusted model & case-mix + MICS models \\
\hline Continuous value & 16,370 & $3,084(18.8)$ & $1,280(7.8)$ & $546(3.3)$ & $0.66(0.62-0.70)$ & $0.95(0.88-1.02)$ \\
\hline$\leq 3.6$ & 431 & $158(36.7)$ & $49(11.4)$ & $10(2.3)$ & $2.05(1.74-2.43)$ & $1.36(1.15-1.62)$ \\
\hline$>3.6$ to $\leq 4.0$ & 1,959 & $464(23.7)$ & $193(9.9)$ & $48(2.5)$ & $1.27(1.14-1.42)$ & $1.08(0.97-1.21)$ \\
\hline$>4.0$ to $\leq 4.5$ & 5,412 & $1,049(19.4)$ & $442(8.2)$ & $161(3.0)$ & 1 (reference) & 1 (reference) \\
\hline$>4.5$ to $\leq 5.0$ & 5,094 & $861(16.9)$ & $360(7.1)$ & $183(3.6)$ & $0.84(0.76-0.91)$ & $1.00(0.91-1.10)$ \\
\hline$>5.0$ to $\leq 5.5$ & 2,572 & $417(16.2)$ & $181(7.0)$ & $114(4.4)$ & $0.75(0.67-0.84)$ & $1.05(0.94-1.19)$ \\
\hline$>5.5$ & 902 & $135(15.0)$ & $55(6.1)$ & $30(3.3)$ & $0.66(0.55-0.78)$ & $1.01(0.84-1.22)$ \\
\hline
\end{tabular}

Case-mix + MICS models adjusted for age, sex, diabetes mellitus, primary insurance, vascular access type, spKt/V, HD treatment time, UF, and cardiovascular risk factors including pre-HD systolic BP, pre-HD diastolic BP, BMI, comorbidities, 13 surrogates of nutritional and/or inflammatory status (albumin, hemoglobin, peripheral WBC, lymphocyte percentage, ferritin, TIBC, calcium, phosphorus, bicarbonate, BUN, iPTH, nPCR, and ESA dose). $n$, number; $\mathrm{CV}$, cardiovascular; HR, hazard ratio. HD, hemodialysis; UF, ultrafiltration; BMI, body mass index; TIBC, total iron binding capacity; BUN, blood urea nitrogen; $\mathrm{iPTH}$, intact parathyroid hormone; $\mathrm{nPCR}$, normalized protein catabolic rate; ESA, erythropoiesis stimulating agent.

tality risk in whites and African-Americans, but not in Hispanics were similar to that in our main analyses (online suppl. Appendix-Fig. S5).

\section{Discussion}

In this study, we examined the differences in the relationship between serum potassium level and all-cause mortality across $3 \mathrm{racial} / \mathrm{ethnic}$ groups of non-Hispanic whites, African-Americans, and Hispanics in a contemporary cohort of over 100,000 incident HD patients who were followed for up to 5 years. We found that Hispanic HD patients tended to be more hyperkalemic than whites and African-Americans. We also observed that hyperkalemia was associated with higher mortality risk in whites and African-American HD patients, but not in Hispanics. Furthermore, in analyses that concurrently examined the association of serum potassium and race/ ethnicity with mortality, compared with Hispanics with 


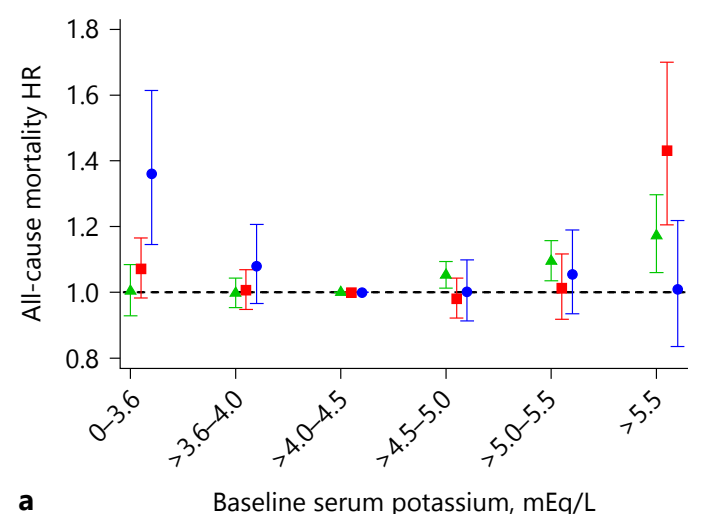

a

Baseline serum potassium, $\mathrm{mEq} / \mathrm{L}$
Fig. 2. Association between serum potassium categories and allcause mortality (a) and cardiovascular mortality (b) across race/ ethnicity in case-mix + MICS models (reference: serum potassium $>4.0$ to $4.5 \mathrm{mEq} / \mathrm{L}$ ). Case-mix + MICS models adjusted for age, sex, $\mathrm{DM}$, primary insurance, vascular access type, spKt/V, HD treatment time, UF, and cardiovascular risk factors including pre-HD systolic BP, pre-HD diastolic BP, BMI, comorbidities, 13 surrogates of nutritional and/or inflammatory status (albumin, hemo-

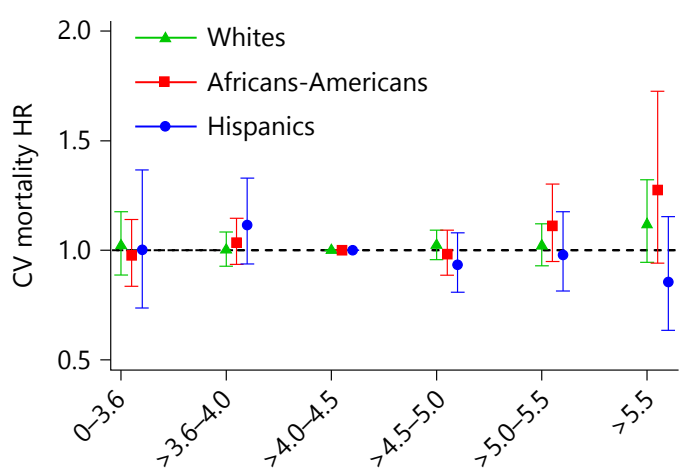

b globin, peripheral WBC, lymphocyte percentage, ferritin, TIBC, calcium, phosphorus, bicarbonate, BUN, iPTH, nPCR, and ESA dose). DM, diabetes mellitus; HD, hemodialysis; UF, ultrafiltration; BMI, body mass index; TIBC, total iron binding capacity; $\mathrm{BUN}$, blood urea nitrogen; iPTH, intact parathyroid hormone; nPCR, normalized protein catabolic rate; ESA, erythropoiesis stimulating agent.
Fig. 3. Association between 18 groups of race/ethnicity and baseline serum potassium with all-cause mortality in case-mix + MICS models (reference: Hispanics with serum potassium $>4.0$ to $4.5 \mathrm{mEq} / \mathrm{L}$ ). Casemix + MICS models adjusted for age, sex, $\mathrm{DM}$, primary insurance, vascular access type, spKt/V, HD treatment time, UF, and cardiovascular risk factors including preHD systolic BP, pre-HD diastolic BP, BMI, comorbidities, 13 surrogates of nutritional and/or inflammatory status (albumin, hemoglobin, peripheral WBC, lymphocyte percentage, ferritin, TIBC, calcium, phosphorus, bicarbonate, BUN, iPTH, nPCR, and ESA dose). DM, diabetes mellitus; HD, hemodialysis; UF, ultrafiltration; BMI, body mass index; TIBC, total iron binding capacity; BUN, blood urea nitrogen; iPTH, intact parathyroid hormone; nPCR, normalized protein catabolic rate; ESA, erythropoiesis stimulating agent.

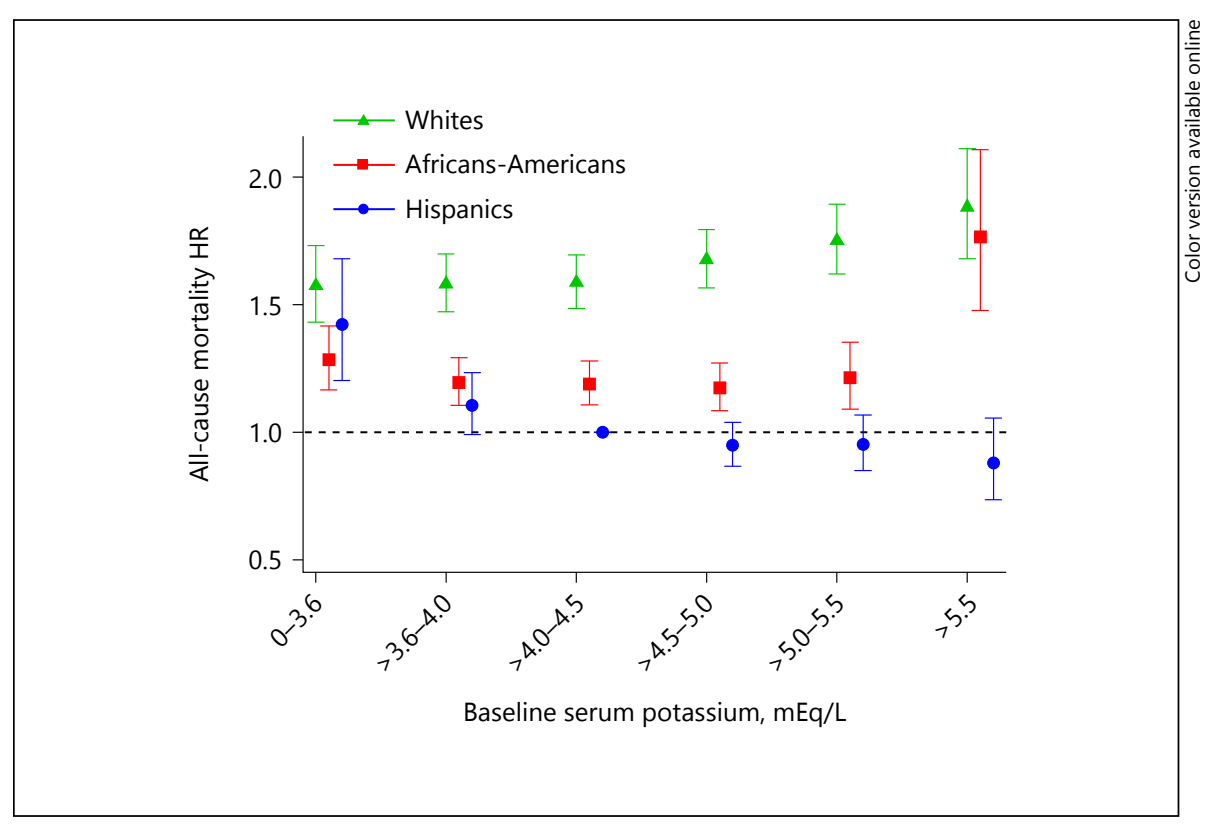

moderate range serum potassium levels ( $>4$ to 4.5 $\mathrm{mEq} / \mathrm{L}$ ), whites and African-Americans across all potassium categories had higher mortality risk, whereas Hispanics with serum potassium levels $\leq 3.6 \mathrm{mEq} / \mathrm{L}$ had higher mortality risk.

Potassium and Mortality across Race/ Ethnicity
To our knowledge, ours is the first study to examine the differences in serum potassium distribution and associations with mortality in HD patients across racial/ ethnic subgroups, including African-Americans, whites, and Hispanics. To date, there has been one study that has 


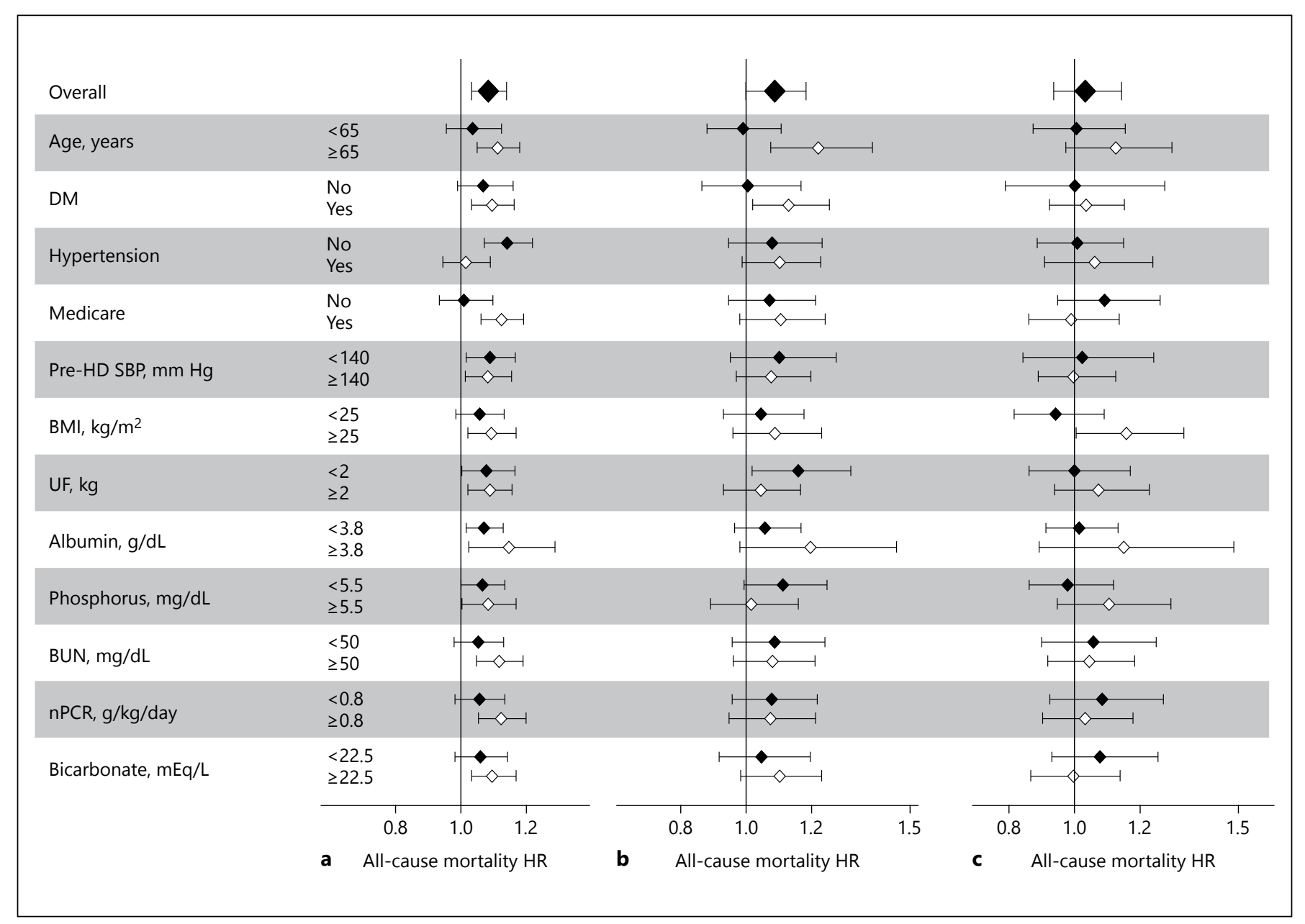

Fig. 4. Association between hyperkalemia (serum potassium $>5.0$ $\mathrm{mEq} / \mathrm{L}$ ) and all-cause mortality (reference: normal serum potassium $>3.6$ to $\leq 5.0 \mathrm{mEq} / \mathrm{L}$ ) across clinically relevant subgroups stratified by race/ethnicity in case-mix + MICS models. Whites (a), African-Americans (b), Hispanics (c). Case-mix + MICS models adjusted for age, sex, DM, primary insurance, vascular access type, spKt/V, HD treatment time, UF, and cardiovascular risk factors including pre-HD systolic BP, pre-HD diastolic BP, BMI, comor-

examined the association between serum potassium levels and mortality across race in pre-dialysis CKD patients [19]. In one study by Hayes et al. [19], white CKD patients had higher baseline serum potassium levels compared to African-Americans, and associations of hyperkalemia with higher mortality risk were observed in white patients only, while associations of hypokalemia with higher mortality were found in both black and white patients. Notably, this study did not include dialysis patients or distinguish between Hispanics vs. non-Hispanics. Another previous study has also examined associations between potassium and mortality in pre-dialysis CKD patients, bidities, 13 surrogates of nutritional and/or inflammatory status (albumin, hemoglobin, peripheral WBC, lymphocyte percentage, ferritin, TIBC, calcium, phosphorus, bicarbonate, BUN, iPTH, nPCR, and ESA dose). DM, diabetes mellitus; HD, hemodialysis; SBP, systolic blood pressure; BMI, body mass index; UF, ultrafiltration; BUN, blood urea nitrogen; $\mathrm{nPCR}$, normalized protein catabolic rate.

and found that African-Americans had significantly lower levels of serum potassium. However, this study did not examine effect modification by race/ethnicity [20].

While the sources of racial/ethnic differences in serum potassium levels remains unclear, it is possible that this may be explained by dietary differences across these groups. According to the National Health and Nutrition Examination Survey data from 1999 to 2002, AfricanAmericans tended to consume less fruits and vegetables and more meat than whites and Hispanics, which may explain the lower serum potassium levels found in this racial/ethnic subgroup $[21,22]$. Despite differences ob- 
served in serum potassium levels, there were no significant differences between Hispanics and whites in the consumption of fruit and vegetables [21]. However, the primary sources of potassium intake in Hispanics were from eggs, whereas in whites this was obtained from legumes, nuts, seeds, dairy products, and beverages [23]. In another study by Kalantar-Zadeh et al. [24], characterizing food consumption in patients initiating $\mathrm{HD}$, it was found that, after receiving dietary instructions, patients independently modified their diet and limited potassium intake to maintain appropriate serum potassium levels. Although Hispanics and non-Hispanics had identical adherence to potassium restriction recommendations [25], the overall dietary modifications may have been more challenging in Hispanic patients due to the impact of cultural differences in food choice [26]. Due to data limitations, we were unable to determine if the higher serum potassium distribution in Hispanics was due to higher intake of fruits and vegetables related to cultural background.

Differences in serum potassium levels may also be impacted by potassium homeostasis. It is well established that African-Americans have lower serum potassium levels that may be attributed to a blunted natriuretic response and lower $\mathrm{Na}^{+}-\mathrm{K}^{+}$ATPase activity $[27,28]$. These may also be related to differences in intracellular potassium content, RAAS activity, and/or intestinal potassium transport. However, since we cannot measure the total body potassium or intracellular potassium in our cohort, we can only speculate what lower serum potassium levels in African-American HD patients represents, and we cannot conclude what effect this might have on membrane potential and excitability, and ultimately on clinical events, including mortality. Increases in excretion or transcellular shifts and alterations in $\mathrm{Na}^{+}-\mathrm{K}^{+}$ATPase activity may be additionally associated with diabetic comorbid conditions [29]. In our cohort, African-Americans had a significantly higher prevalence of diabetes comorbidity. Although these mechanistic differences may explain why African-Americans had lower levels of serum potassium overall, in analyses adjusted for diabetes as well as across diabetes strata, we did not see significant differences in the associations of hyperkalemia with mortality outcomes.

Conversely, in our cohort Hispanics had the highest prevalence of diabetes comorbidity, but had the highest levels of baseline serum potassium. Associations of hyperkalemia with mortality were attenuated in Hispanic diabetic patients; however, there were no significant interactions between race and diabetes in the associations of hyperkalemia with mortality. To date, there is a lack

Potassium and Mortality across Race/ Ethnicity of data examining differences in biological mechanisms of potassium levels in Hispanic vs. non-Hispanic patients. Additional information on factors that affect membrane excitability in this racial/ethnic group are needed to better understand if these factors can explain differences in mortality outcomes associated with the same serum potassium levels.

Racial/ethnic genetic differences may explain the differential association between hyperkalemia and mortality risk across racial/ethnic groups. Indeed, a mutational analysis study conducted in non-CKD patients has identified distinct cardiac potassium channel variants related to higher risk of sudden cardiac death across racial/ethnic groups, and further study in the kidney disease population is needed [30].

Patients who chronically consume a high potassium diet may develop potassium tolerance, and they may no longer be susceptible to the adverse sequelae of hyperkalemia. In a large national cohort study of veterans, patients with CKD had a higher frequency of hyperkalemic events as compared with non-CKD patients. However, the mortality predictability of hyperkalemia was inversely related to the CKD stage, presumably due to the development of potassium tolerance and less subsequent susceptibility to cardiac toxicity due to chronic exposure to high potassium levels [3]. It is possible that Hispanic patients who are exposed to a high potassium diet over a lifetime may develop such potassium tolerance and thereby have a lower risk of mortality while in the hyperkalemic state. It may also explain why Hispanic patients with lower serum potassium level have a higher mortality risk, since it may be a departure from a normative healthy state.

A systematic review has shown that Hispanics have a lower cardiovascular mortality than non-Hispanic whites due to the differences in diet patterns that improve the endothelial function and physical activity that modifies cardiovascular risk factors [31]. Although Hispanics have a greater prevalence of cardiovascular risk factors such as diabetes, abdominal obesity, and hypertriglyceridemia [32], several studies have demonstrated that Hispanics with CKD and ESRD had a lower incidence of cardiovascular events compared with non-Hispanic whites [12, 33]. It is possible that fewer cardiovascular events account for lower cardiovascular mortality in Hispanics with higher serum potassium, but further study is needed to determine the mechanism. To our knowledge, this is the first study to evaluate racial/ethnic differences in the relationship between serum potassium levels and mortality in a large nationally representative, contemporary cohort 
of incident HD patients with detailed capture of sociodemographic, comorbidity, and laboratory data. However, our study has several limitations. First, Hispanics may be heterogeneous in terms of cultural backgrounds and countries of origin, which may have implications on serum potassium concentrations $[34,35]$. We were unable to distinguish Hispanic subpopulations, which may be white or black race. It has been shown that Hispanic subgroups in the US originating from different countries including Mexico, the Caribbean, and Central and South America have differences in the consumption of fruits, vegetables, meat, and fats [36]. Potassium intake also differed according to individual and/or neighboring socioeconomic status [37]. We did not have data on dietary potassium intake. In addition, information on residual renal function was only available for one third of our cohort. Analyses adjusting for potential confounding due to residual renal function may be inherent to selection biases, and future studies investigating the impact of residual renal function on the racial/ethnic differences in serum potassium mortality associations are warranted. We also lacked data on certain dialysis treatment characteristics that may influence serum potassium concentrations such as use of RAAS inhibitors and diuretics and dialysate potassium concentration. While one observational study has shown that higher dialysate potassium bath was associated with higher mortality risk in HD patients with hyperkalemia [4], another study showed no difference in cardiac death between dialysate potassium bath and serum potassium levels even amongst hyperkalemic HD patients [38]. Given the observational nature of this cohort analysis, our study is limited to adjusting for confounders that were measured in the dataset. We thereby cannot exclude the possibility of potential residual confounding.

In conclusion, our study has shown that Hispanic HD patients tend to have higher serum potassium levels as compared to African-American and white HD patients. While African-Americans and whites with higher potassium levels experienced higher death risk, this was not observed in Hispanic HD patients. Potential mechanisms underlying differences in serum potassium distributions and associations with mortality may be related to socio-cultural influences such as high potassium tolerance related to a chronically high potassium diet, or potassium adaptation related to $\mathrm{racial} / \mathrm{ethnic}$ genetic differences. Further studies are needed to determine the possible causal mechanisms underlying the survival advantage observed in hyperkalemic Hispanic HD patients.

\section{Disclosure Statement}

K.K.-Z. has received honoraria and/or support from Abbott, Abbvie, Alexion, Amgen, American Society of Nephrology, Astra-Zeneca, AVEO, Chugai, DaVita, Fresenius, Genetech, Haymarket Media, Hospira, Kabi, Keryx, National Institutes of Health, National Kidney Foundation, Relypsa, Resverlogix, Sanofi, Shire, Vifor, ZSPharma, and was the medical director of DaVita Harbor-UCLA/ MFI in Long Beach, CA during 2007-2012. C.P.K. has received honoraria from Abbott Nutrition, Sanofi-Aventis, Relypsa and ZS Pharma. Other authors have not declared any conflicts of interest.

\section{Acknowledgments}

The study was supported by Dr. Kamyar Kalantar-Zadeh's research grants from the National Institute of Diabetes, Digestive and Kidney Disease (NIDDK) of the National Institute of Health (NIH; K24-DK09141, R01-DK078106, and R01-DK095668), and philanthropic grants from Mr. Harold Simmons, Mr. Louis Chang, Dr. Joseph Lee, and AVEO, Inc., Dr. Kovesdy's NIH (NIDDK) grants R01-DK096920 and U01-DK102163, and Dr. Connie M. Rhee NIH (NIDDK) grant K23-DK102903. Dr. Elani Streja is supported by a career development award from the Office of Research and Development of the Department of Veterans Affairs (IK2CX001266-01). Dr. Yoshitsugu Obi is supported by the Uehara Memorial Foundation Research Fellowship for Research Abroad.

\section{Author's Roles}

Study design: T.K., E.S., C.M.R., C.P.K., and K.K.-Z. Data analysis: T.K. and E.S. Data interpretation: T.K., C.M.R., E.S., Y.O., J.A.C., A.R.T., V.A.R., M.S., C.P.K., and K.K.-Z. Drafting manuscript: T.K., C.M.R., E.S., Y.O., J.A.C., A.R.T., V.A.R., M.S., C.P.K., and K.K.-Z. Approving final version of manuscript: T.K., C.M.R., E.S., Y.O., J.A.C., A.R.T., V.A.R., M.S., C.P.K., and K.K.-Z. Each author contributed important intellectual content during manuscript drafting and accepts accountability for the overall work by ensuring that questions pertaining to the accuracy or integrity of any portion of the work are appropriately investigated and resolved. K.K.-Z. takes responsibility for the integrity of the data analysis.

References

Am J Nephrol 2017;45:509-521 DOI: $10.1159 / 000475997$
1 Palmer BF: Regulation of potassium homeostasis. Clin J Am Soc Nephrol 2015; 10:1050-1060.

2 Pani A, Floris M, Rosner MH, Ronco C: Hyperkalemia in hemodialysis patients. Semin Dial 2014;27:571-576.

3 Einhorn LM, Zhan M, Hsu VD, et al: The frequency of hyperkalemia and its significance in chronic kidney disease. Arch Intern Med 2009;169:1156-1162.

4 Kovesdy CP, Regidor DL, Mehrotra R, et al: Serum and dialysate potassium concentrations and survival in hemodialysis patients. Clin J Am Soc Nephrol 2007;2:999-1007.

Kim/Rhee/Streja/Soohoo/Obi/Chou/ Tortorici/Ravel/Kovesdy/Kalantar-Zadeh 
5 Genovesi S, Valsecchi MG, Rossi E, et al: Sudden death and associated factors in a historical cohort of chronic haemodialysis patients. Nephrol Dial Transplant 2009;24: 2529-2536.

6 Collins AJ, Foley RN, Gilbertson DT, Chen SC: United States Renal Data System public health surveillance of chronic kidney disease and end-stage renal disease. Kidney Int Suppl (2011) 2015;5:2-7.

7 Turban S, Miller ER 3rd, Ange B, Appel LJ: Racial differences in urinary potassium excretion. J Am Soc Nephrol 2008;19:1396-1402.

8 Cavallari LH, Fashingbauer LA, Beitelshees $\mathrm{AL}$, et al: Racial differences in patients' potassium concentrations during spironolactone therapy for heart failure. Pharmacotherapy 2004;24:750-756.

9 United States Renal Data System: 2014 USRDS Annual Data Report: Epidemiology of Kidney Disease in the United States. Bethesda, National Institutes of Health, $\mathrm{Na}$ tional Institute of Diabetes and Digestive and Kidney Diseases, 2014.

10 Kucirka LM, Grams ME, Lessler J, et al. Association of race and age with survival among patients undergoing dialysis. JAMA 2011; 306:620-626.

11 Yan G, Norris KC, Yu AJ, et al: The relationship of age, race, and ethnicity with survival in dialysis patients. Clin J Am Soc Nephrol 2013;8:953-961.

12 Murthy BV, Molony DA, Stack AG: Survival advantage of Hispanic patients initiating dialysis in the United States is modified by race. J Am Soc Nephrol 2005;16:782-790.

13 Frankenfield DL, Rocco MV, Roman SH, McClellan WM: Survival advantage for adult Hispanic hemodialysis patients? Findings from the end-stage renal disease clinical performance measures project. J Am Soc Nephrol 2003; 14:180-186.

14 Arce CM, Goldstein BA, Mitani AA, Winkelmayer WC: Trends in relative mortality between Hispanic and non-Hispanic whites initiating dialysis: a retrospective study of the US Renal Data System. Am J Kidney Dis 2013;62: 312-321.

15 Daugirdas JT: Physiologic principles and urea kinetic modeling; in Daugirdas JT, Blake PG, Ing TS (eds): Handbook of Dialysis, ed 5. Philadelphia, Lippincott Williams \& Wilkins, 2014, pp 59-60.
16 Hemodialysis Adequacy 2006 Work Group: Clinical practice guidelines for hemodialysis adequacy, update 2006. Am J Kidney Dis 2006;48(suppl 1):S2-S90.

17 Mosteller RD: Simplified calculation of bodysurface area. N Engl J Med 1987;317:1098.

18 Fine JP, Gray RJ: A proportional hazards model for the subdistribution of a competing risk. J Am Stat Assoc 1999;94:496-509.

19 Hayes J, Kalantar-Zadeh K, Lu JL, Turban S, Anderson JE, Kovesdy CP: Association of hypo- and hyperkalemia with disease progression and mortality in males with chronic kidney disease: the role of race. Nephron Clin Pract 2012;120:c8-c16.

20 Nakhoul GN, Huang H, Arrigain S, et al: Serum potassium, end-stage renal disease and mortality in chronic kidney disease. Am J Nephrol 2015;41:456-463.

21 Casagrande SS, Wang Y, Anderson C, Gary TL: Have Americans increased their fruit and vegetable intake? The trends between 1988 and 2002. Am J Prev Med 2007;32:257-263.

22 Chan KH, Chacko SA, Song Y, et al: Genetic variations in magnesium-related ion channels may affect diabetes risk among African American and Hispanic American women. J Nutr 2015; 145:418-424.

23 O'Neil CE, Nicklas TA, Keast DR, Fulgoni VL: Ethnic disparities among food sources of energy and nutrients of public health concern and nutrients to limit in adults in the United States: NHANES 2003-2006. Food Nutr Res 2014;58:15784.

24 Kalantar-Zadeh K, Kopple JD, Deepak S, Block D, Block G: Food intake characteristics of hemodialysis patients as obtained by food frequency questionnaire. J Ren Nutr 2002;12: 17-31.

25 Morales Lopez C, Burrowes JD, Gizis F, Brommage D: Dietary adherence in Hispanic patients receiving hemodialysis. J Ren Nutr 2007;17:138-147.

26 Shepherd R: Influences on food choice and dietary behavior. Forum Nutr 2005;57:36-43.

27 Luft FC, Grim CE, Fineberg N, Weinberger MC: Effects of volume expansion and contraction in normotensive whites, blacks, and subjects of different ages. Circulation 1979; 59:643-650

28 Suh A, DeJesus E, Rosner K, et al: Racial differences in potassium disposal. Kidney Int 2004;66:1076-1081.
29 Gennari FJ: Disorders of potassium homeostasis. Hypokalemia and hyperkalemia. Crit Care Clin 2002;18:273-288, vi.

30 Ackerman MJ, Tester DJ, Jones GS, Will ML, Burrow CR, Curran ME: Ethnic differences in cardiac potassium channel variants: implications for genetic susceptibility to sudden cardiac death and genetic testing for congenital long QT syndrome. Mayo Clin Proc 2003;78: 1479-1487.

31 Cortes-Bergoderi M, Goel K, Murad MH, et al: Cardiovascular mortality in Hispanics compared to non-Hispanic whites: a systematic review and meta-analysis of the Hispanic paradox. Eur J Intern Med 2013;24:791-799.

32 Ford ES, Giles WH, Dietz WH: Prevalence of the metabolic syndrome among US adults: findings from the third National Health and Nutrition Examination Survey. JAMA 2002; 287:356-359.

33 Peralta CA, Shlipak MG, Fan D, et al: Risks for end-stage renal disease, cardiovascular events, and death in Hispanic versus non-Hispanic white adults with chronic kidney disease. J Am Soc Nephrol 2006;17:2892-2899.

34 Daviglus ML, Talavera GA, Aviles-Santa ML, et al: Prevalence of major cardiovascular risk factors and cardiovascular diseases among Hispanic/Latino individuals of diverse backgrounds in the United States. JAMA 2012; 308:1775-1784.

35 Borrell LN, Crawford ND: All-cause mortality among Hispanics in the United States: exploring heterogeneity by nativity status, country of origin, and race in the National Health Interview Survey-linked mortality files. Ann Epidemiol 2009;19:336-343.

36 Siega-Riz AM, Sotres-Alvarez D, Ayala GX, et al: Food-group and nutrient-density intakes by Hispanic and Latino backgrounds in the Hispanic Community Health Study/ Study of Latinos. Am J Clin Nutr 2014;99: 1487-1498.

37 Yi SS, Ruff RR, Jung M, Waddell EN: Racial/ ethnic residential segregation, neighborhood poverty and urinary biomarkers of diet in New York City adults. Soc Sci Med 2014;122: 122-129.

38 Pun PH, Lehrich RW, Honeycutt EF, Herzog CA, Middleton JP: Modifiable risk factors associated with sudden cardiac arrest within hemodialysis clinics. Kidney Int 2011;79:218227. 